Published in final edited form as:

Chembiochem. 2020 October 01; 21(19): 2764-2767. doi:10.1002/cbic.202000260.

\title{
A Step Toward Molecular Evolution of RNA: Ribose Binds to Prebiotic Fatty Acid Membranes, and Nucleosides Bind Better Than Individual Bases Do
}

\author{
Dr. M. Xue ${ }^{a}$, Affiliate Prof. R.A. Black ${ }^{a}$, C.E. Cornell ${ }^{a}$, Prof. G.P. Drobny ${ }^{a}$, Prof. S.L. Keller ${ }^{a}$ \\ aDepartment of Chemistry, University of Washington, Seattle WA 98195 USA
}

\begin{abstract}
A major challenge in understanding how biological cells arose on the early Earth is explaining how RNA and membranes originally co-localized. We propose that the building blocks of RNA (nucleobases and ribose) bound to self-assembled prebiotic membranes. We have previously demonstrated that the bases bind to membranes composed of a prebiotic fatty acid, but evidence for the binding of sugars has remained a technical challenge. Here, we use pulsed field gradient nuclear magnetic resonance to demonstrate that ribose and other sugars bind to membranes of decanoic acid. Moreover, binding of some bases is strongly enhanced when they are linked to ribose to form a nucleoside or - with the addition of phosphate—a nucleotide. This enhanced binding could have played a role in the molecular evolution leading to the production of RNA.
\end{abstract}

\section{Graphical Abstract}

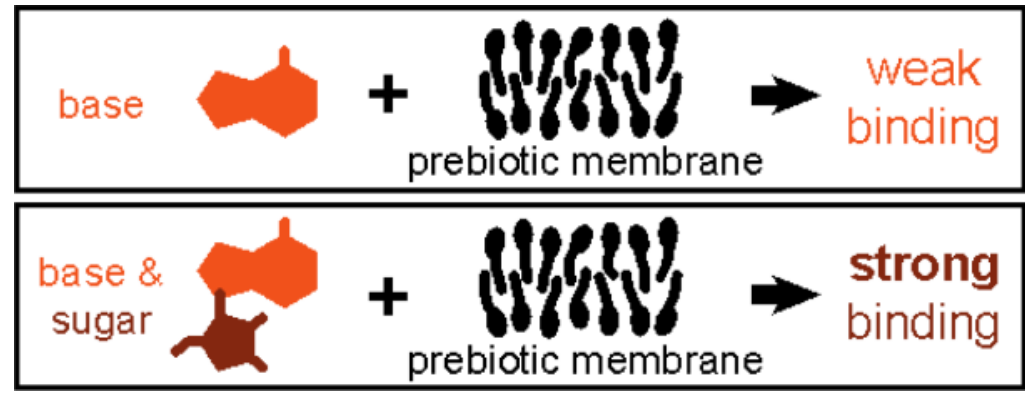

Prior to their polymerization into RNA, nucleobases and ribose may have bound to self-assembled, prebiotic membranes. We found that ribose binds more strongly to fatty acid membranes than bases do. Linking ribose to a nucleobase to form a nucleoside, a subunit of RNA, strengthens the nucleobase's binding.

\section{Keywords}

membrane; origin of life; prebiotic; protocell; RNA

blackr5@uw.edu.

Experimental section

Experimental details are described in the Supporting Information. 
At its simplest, the first protocell on Earth was a bag of information. The bag would have been a self-assembled membrane of fatty acids. ${ }^{[1-3]}$ RNA or a similar molecule would have encoded information. ${ }^{[4]}$ Major challenges persist in explaining how sugars and bases, the building blocks of RNA, could have locally reached concentrations high enough to support RNA synthesis, and, in turn, how RNA co-localized with membranes. ${ }^{[5]}$ One possible answer to both challenges is that the building blocks of RNA bound to fatty acid membranes. ${ }^{[6,7]}$

We previously found that several sugars protect fatty acid vesicles against destabilizing effects of high $\mathrm{NaCl}$ concentrations, ${ }^{[6]}$ but whether these or other sugars bound to the vesicles remained an open question. Several bases also protected the vesicles against high salt, and we used a filtration assay to show that these bases bound to fatty acid surfaces. These prior studies did not address whether more complex building blocks of RNA such as nucleosides (the products of sugars and bases) or nucleotides (the products of nucleosides and a phosphate) bind to fatty acid membranes.

Here we use pulsed field gradient nuclear magnetic resonance (diffusion NMR) to test for binding of sugars, bases, nucleosides, and a nucleotide to fatty acid membranes. Small, unbound molecules diffuse quickly in solution; when they bind to the surfaces of a fatty acid membrane, their apparent diffusion coefficient decreases. ${ }^{[8,9]}$ Evaluating binding by diffusion NMR avoids shortcomings of several other techniques. Our emphasis is on prebiotically plausible molecules. Fatty acids with 10 carbons (decanoic acid) are short enough to have been available on the early Earth ${ }^{[10]}$ and long enough to self-assemble into membranes. ${ }^{[11]}$ These membranes are directly visible as micron-scale, free-floating vesicles in solution (Fig. 1). The bases and sugars we use are also plausibly prebiotic. ${ }^{[12-15]}$

Our first major result is that sugars bind more strongly to fatty acid membranes than bases do. Two sugars present in information-carrying polymers are ribose (found in RNA) and deoxyribose (found in DNA). Minor changes in these structures lead to an array of diastereomers (e.g. xylose). When these three sugars are free in solution, their motion is characterized by a single, fast diffusion coefficient, which produces a single slope on a plot of NMR echo intensity vs. field strength (Fig. 2, Table S1). When the sugars are in the presence of decanoic acid vesicles, a second, shallow slope appears (Fig. 2, Table 1, Fig. S1, S2). Two conditions must be met for the second slope to be clearly distinguishable. First, exchange of the sugars between the solution and the vesicle surface must be near to or slower than the diffusion time (the time between gradient pulses) of $0.3 \mathrm{~s}$. Second, there must be relatively strong binding of the sugars to the surface, such that their diffusion coefficients decrease by at least an order of magnitude. Roughly $5 \%$ of the sugar molecules are bound to vesicles (by applying equations 2 and 3 in the methods). The amount of ribose bound increases with the total concentration of ribose over the range we tested (Fig. S3, Table S2). The vesicle solution likely also contains decanoic acid micelles, to which test compounds might bind. Those micelles, which have a diameter of only $\sim 2 \mathrm{~nm}$, are not the focus of our current study. Previous researchers found diffusion coefficients of micelles to be $\sim 2 \times 10^{-10} \mathrm{~m}^{2} / \mathrm{s},{ }^{[16]}$ the same order of magnitude as $D_{\text {free }}$ in Table 1 . Therefore, test compounds might bind to fatty acid surfaces (a term that encompasses micelles as well as vesicles) at fractions higher than $f_{\text {bound }}$ in Table 1 , which pertains to only vesicles. Binding 
of sugars to membranes is consistent with (but could not have been predicted by) our previously reported observations that ribose (and, to a lesser extent, xylose) protects decanoic acid vesicles against flocculation due to $\mathrm{NaCl}^{[6]}$

Sugars (all of which were D-enantiomers), bases, nucleosides, and nucleotides were dissolved in $50 \mathrm{mM}$ decanoic acid as described in the Methods. For five samples, data were re-analyzed using the alternate peaks shown; all other peaks are identified in figures. Quoted uncertainties in diffusion coefficients represent only the uncertainty of the fit. Sample-tosample differences in $D_{\text {bound }}$ can vary by as much as $77 \%$ of the average (i.e. in the case of cytosine). However, as demonstrated in the Methods, the exact value of $D_{\text {bound }}$ has very little influence on the fraction bound, which is similar between duplicates. Equations for percent bound values and discussion of the uncertainties in their fits (which are $\sim 10 \%$ of their values) appear in the Methods. Corresponding diffusion coefficients for all compounds in the absence of decanoic acid are shown in Table S1.

When molecules initially in solution bind to membranes, the average distance between the molecules can decrease significantly, even when only a fraction of the molecules bind. For example, in a solution in which $\sim 5 \%$ of $15 \mathrm{mM}$ sugar is bound to membranes, the average distance between sugar molecules in solution is $49 \AA$. To calculate the maximum distance between sugars bound to a membrane, we need an upper bound of the available membrane surface, which comes from assuming that every decanoic acid molecule above its critical micelle concentration of $\sim 15 \mathrm{mM}^{[8]}$ self-assembles into a vesicle. We also assume that sugars pass freely through fatty acid membranes, ${ }^{[17,18]}$ such that the interior surface of the vesicle is as accessible as the exterior. Then, when $5 \%$ of the sugar binds to the membranes, the average distance between the molecules is, at maximum, $37 \AA$. This value is less than 49 $\AA$, the distance between sugars in solution. The full calculation is found in the supplement.

The strong binding of the sugars to fatty acid membranes, shown in Fig. 2, is in contrast to the weak binding of most of the bases, seen in Fig. 3. Only two of the bases we investigated (cytosine and uracil) bound strongly enough to decanoic acid vesicles to produce a second slope by diffusion NMR. The observation that sugars bind to fatty acid membranes more strongly than bases do leads directly to the question of whetherattaching a sugar to a base improves the binding of the base. Stated another way, could the membrane have selected for nucleosides, prior to their incorporation in RNA?

Our second major result is that nucleosides, the products of joining a base to a sugar, do have greater affinity for vesicles than bases alone. For example, adenosine produces two diffusion coefficients in the presence of decanoic acid vesicles, indicating strong binding (Fig. 4), whereas adenine does not (Fig. 3). Similarly, the nucleoside uridine shows stronger binding than its base, uracil. These results led us to ask whether the mechanism of nucleoside binding is similar to that of ribose. We found that the average residence times for both bound and free ribose were about 8-fold greater than for bound and free adenosine (Fig. S4, Table S3). Therefore ribose appears to interact with the vesicle surface more strongly than adenosine. One possible explanation for this difference is that the five-membered ring conformation of ribose, which is locked in when it is part of a nucleoside, binds less strongly than the six- membered ring or straight-chain forms available to free ribose. Another 
possible explanation is that the number of hydroxyl groups available for interaction differs between the nucleoside and ribose.

Enhanced binding of the composite molecule relative to the base is retained when a phosphate group is added to the adenosine to produce adenosine monophosphate, a nucleotide (Fig. 4). (RNA is a string of nucleotides.) The observation that a nucleoside and nucleotide bind roughly equally to fatty acid vesicles suggests that the nucleotide may orient with its negatively-charged phosphate group away from the negatively-charged fatty acid headgroups, as would be expected. Alternatively, attenuation of the electrostatic interaction between the nucleotide's phosphate and the headgroups by the phosphate and $\mathrm{NaCl}$ in the solution may enable additional orientations.

In summary, we find that simple sugars bind to prebiotic, fatty acid membranes and that linking a sugar to a base (as in a nucleoside) or to a base and a phosphate (as in a nucleotide) can result in stronger binding than of bases alone. This means that an individual building block of RNA binds less well to membranes than a compound formed by linking building blocks together. This result could partially explain how molecular selection for nucleosides and nucleotides began. Specifically, the formation of nucleosides and/or nucleotides may have been required in order to achieve co-localization of all of the building blocks of RNA and membranes. An intriguing possibility that follows from this scenario is that the binding of sugars and bases oriented them in a way to overcome some of the known difficulties of nucleoside formation. ${ }^{[19]}$ Overall, these results complement our previous discovery that amino acids bind to fatty acid vesicles ${ }^{[8]}$ to show that all of the major building blocks of protocells could have spontaneously assembled into a single unit.

\section{Supplementary Material}

Refer to Web version on PubMed Central for supplementary material.

\section{Acknowledgements}

The Keller and Drobny laboratories were supported by NASA grant NNX17AK86G (Exobiology). G.P.D. also acknowledges support from NSF MCB-1715123 and NIH RO1-GM109417.

\section{References}

[1]. Deamer D, Dworkin JP, Sandford SA, Bernstein MP, Allamandola LJ, Astrobiology 2002, 2, 371. [PubMed: 12593777]

[2]. Morigaki K, Walde P, Curr. Opin. Coll. Int. Sci 2007, 12, 75.

[3]. Lawless J, Yuen G, Nature 1979, 282, 396.

[4]. Szostak JW, J. Syst. Chem 2012, 3, 2-1.

[5]. Ruiz-Mirazo K, Briones C, de la Escosura A, Chem. Rev 2014, 114, 285. [PubMed: 24171674]

[6]. Black RA, Blosser MC, Stottrup BL, Tavakley R, Deamer DW, Keller SL, Proc. Natl. Acad. Sci. U.S.A 2013, 110, 13272. [PubMed: 23901105]

[7]. Black RA, Blosser MC, Life 2016, 6, 33.

[8]. Cornell CE, Black RA, Xue M, Litz HE, Ramsay A, Gordon M, Mileant A, Cohen ZR, Williams JA, Lee KK, Drobny GP, Keller SL, Proc. Natl. Acad. Sci. U.S.A 2019, 116, 17239. [PubMed: 31405964]

[9]. Mayer C, Schreiber U, Dávila MJ, Orig. Life. Evol. Biosph 2015, 45, 139. [PubMed: 25716918] 
[10]. Naraoka H, Shimoyama A, Harada K, Orig. Life. Evol. Biosph 1999, 29, 187. [PubMed: 10391772]

[11]. Namani T, Walde P, Langmuir 2005, 21, 6210. [PubMed: 15982022]

[12]. Callahan MP, Smith KE, Cleaves HJ, Ruzicka J, Stern JC, Glavin DP, House C, Dworkin JP, Proc. Natl. Acad. Sci. U.S.A 2011, 108, 13995. [PubMed: 21836052]

[13]. Luisi PL, The Emergence of Life, Cambridge University Press, Cambridge, UK, 2006, pp. 44-49.

[14]. Ricardo A, Carrigan MA, Olcott AN, Benner SA, Science 2004, 303, 196. [PubMed: 14716004]

[15]. Lambert JB, Gurusamy-Thangavelu SA, Ma K, Science 2010, 327, 984. [PubMed: 20167782]

[16]. Lewis C, Hughes BH, Vasquez M, M. Wall A, Northrup VL, Witzleb TJ, Billiot EJ, Fang Y, Billiot FH, Morris KF, J Surfact Deterg 2016 19,1175.

[17]. Sacerdote MG, Szostak JW, Proc. Natl. Acad. Sci. U.S.A 2005, 102, 6004. [PubMed: 15831588]

[18]. Wei C, Pohorille A, Astrobiology 2013, 13, 1. [PubMed: 23252378]

[19]. Sutherland JD, Cold Spring Harb. Perspect. Biol 2010, 2;a005439. [PubMed: 20452951] 
A. Decanoic acid vesicle

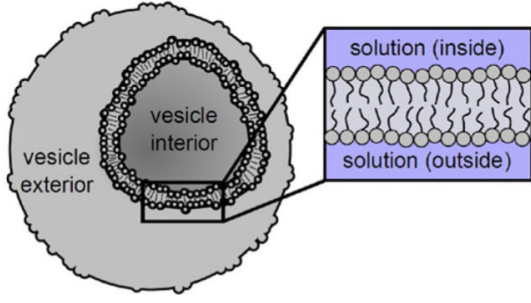

B. Image

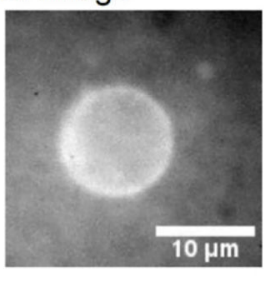

C. Structure

OH $0=$ polar

$\sum_{\text {nonpolar }}$

$\sum$ tail

Figure 1.

A. Cut-away schematic of a vesicle, exposing a self-assembled membrane of fatty acids. B. Fluorescence micrograph of a typical decanoic acid vesicle labeled with rhodamine $6 \mathrm{G}$, floating freely in a $\mathrm{D}_{2} \mathrm{O}$ solution. C. Structure of decanoic acid. 

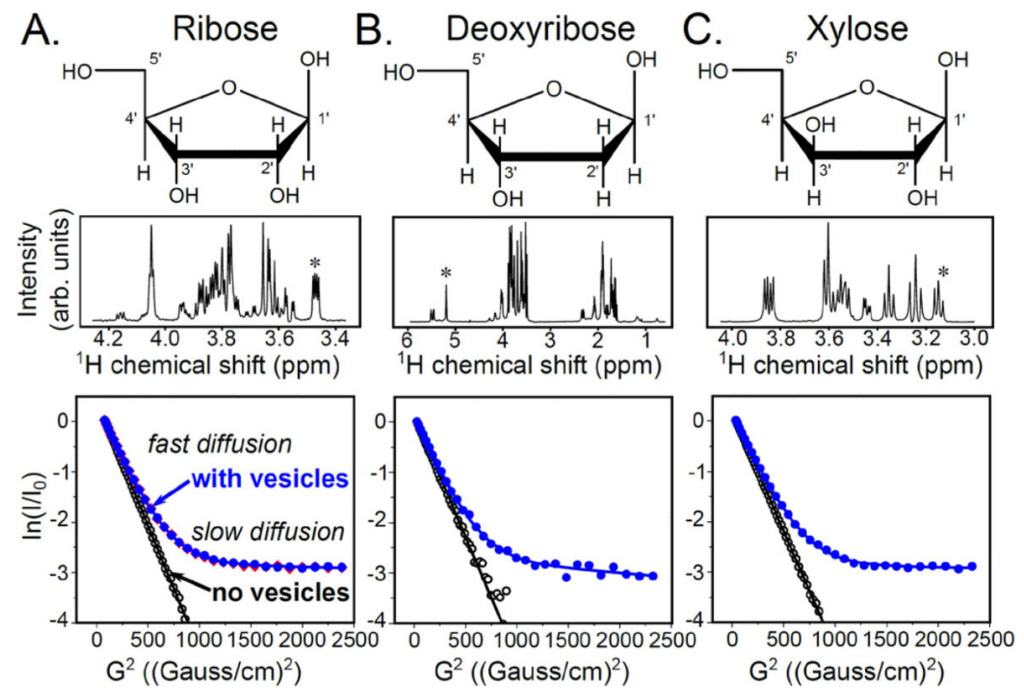

Figure 2.

The sugars in RNA and DNA (A, ribose and B, deoxyribose), and a diastereomer (C, xylose) bind to fatty acid vesicles. Top panels show the structures of the sugars as 5-member rings (which interconvert with 6-member rings and linear chains in solution). Middle panels show ${ }^{1} \mathrm{H}$ NMR spectra (with an asterisk $(*)$ above the main peak analyzed by diffusion NMR). Bottom panels show plots of the square of the NMR magnetic field gradient strength $\left(\mathrm{G}^{2}\right.$, where $\mathrm{G}$ is $\mathrm{dB} / \mathrm{dz}$ in units of Gauss/cm and B is the magnetic field), vs. the normalized peak intensity ( $\ln \mathrm{I} / \mathrm{I}_{0}$ ). Experiments were conducted with $15 \mathrm{mM}$ sugar in the absence (open, black symbols) or presence (closed, blue symbols) of $50 \mathrm{mM}$ deuterated decanoic acid. Ribose samples (red and blue closed symbols) were evaluated twice. 

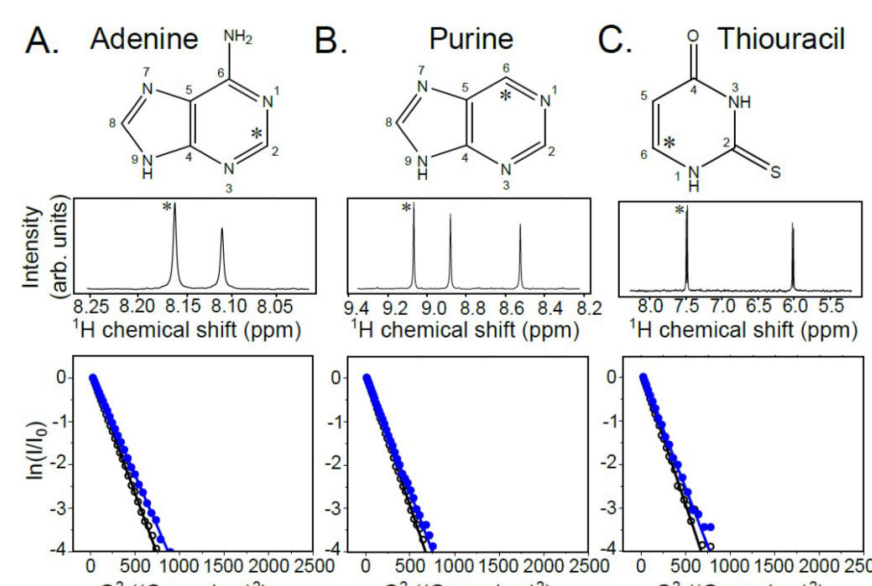

Hemical shift (p.5
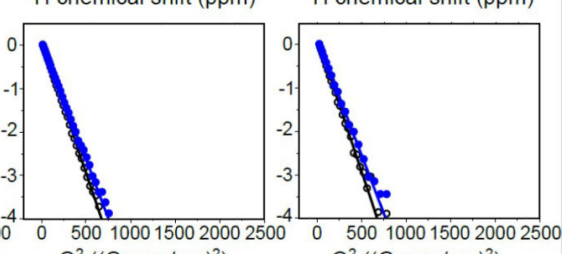
$\mathrm{G}^{2}\left((\mathrm{Gauss} / \mathrm{cm})^{2}\right)$ $\mathrm{G}^{2}\left((\mathrm{Gauss} / \mathrm{cm})^{2}\right)$ $\mathrm{G}^{2}\left((\mathrm{Gauss} / \mathrm{cm})^{2}\right)$

D. $\mathrm{NH}_{2}$ Cytosine

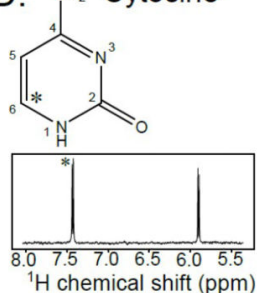

E. o Uracil
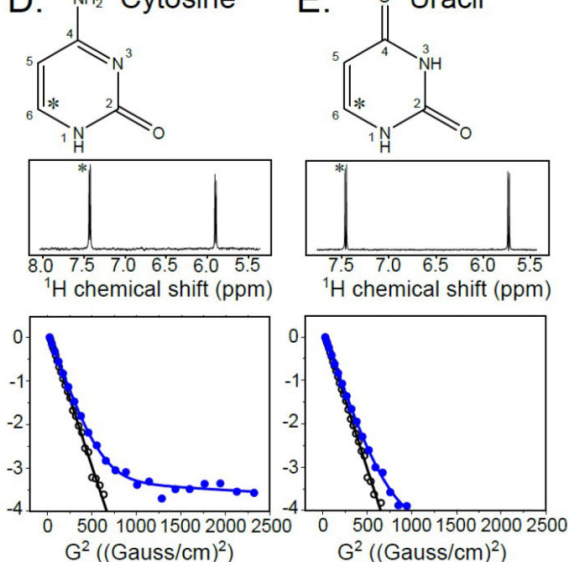

Figure 3.

Among the five bases shown, two bind strongly to fatty acid vesicles. Top panels show structures of the bases: adenine (A), purine (B), thiouracil (C), cytosine (D), and uracil (E). Middle panels show ${ }^{1} \mathrm{H}$ NMR spectra (with an asterisk (*) above the main peak analyzed by diffusion NMR). Bottom panels show plots of the square of NMR magnetic field gradient strength $\left(\mathrm{G}^{2}\right)$ vs. normalized peak intensity $\left(\ln \mathrm{I} / \mathrm{I}_{0}\right)$. Experiments were conducted with 2 $\mathrm{mM}$ of the base in the absence (open, black symbols) or presence (closed, blue symbols) of $50 \mathrm{mM}$ deuterated decanoic acid. 

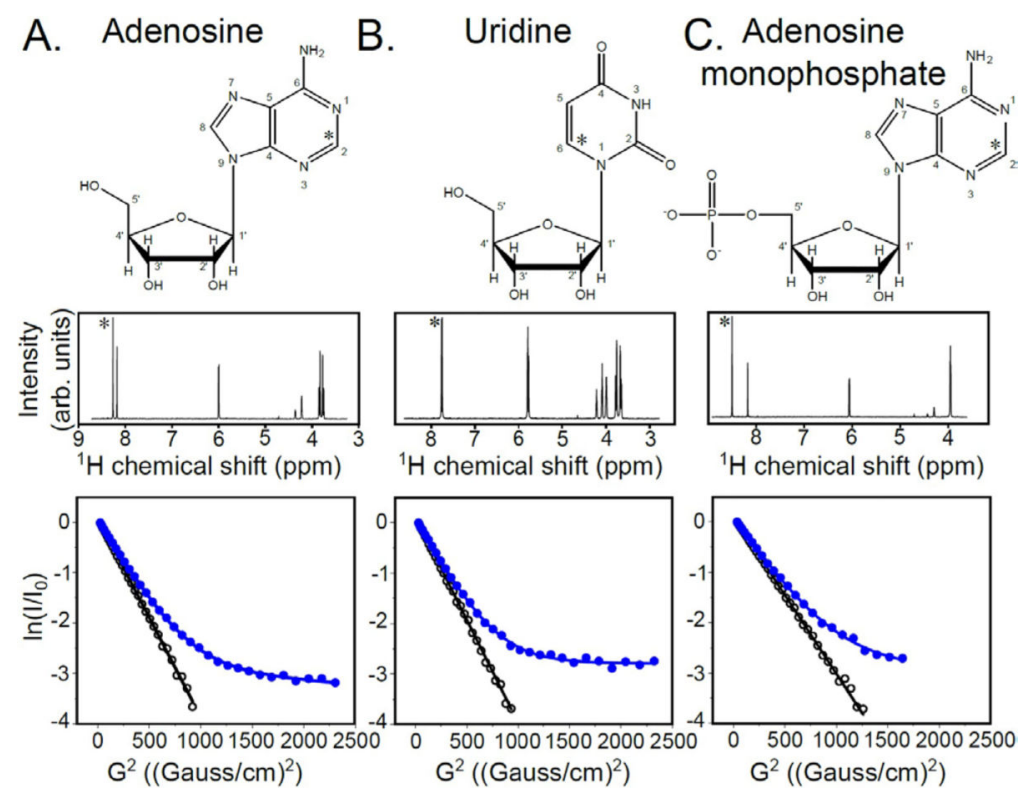

Figure 4.

When bases are covalently bound to a sugar (with or without a phosphate), they bind strongly to fatty acid vesicles. Top panels show structures of two nucleosides (adenosine (A) and uridine (B)) and a nucleotide (adenosine monophosphate $(\mathrm{C})$ ). Middle panels show ${ }^{1} \mathrm{H}$ NMR spectra (with an asterisk (*) above the main peak analyzed by diffusion NMR).

Bottom panels show plots of the square of NMR magnetic field gradient strength $\left(\mathrm{G}^{2}\right)$, vs. normalized peak intensity $\left(\ln \mathrm{I} / \mathrm{I}_{0}\right)$. Experiments were conducted with $2 \mathrm{mM}$ of the nucleoside or nucleotide in the absence (open, black symbols) or presence (closed, blue symbols) of $50 \mathrm{mM}$ deuterated decanoic acid. 
로을

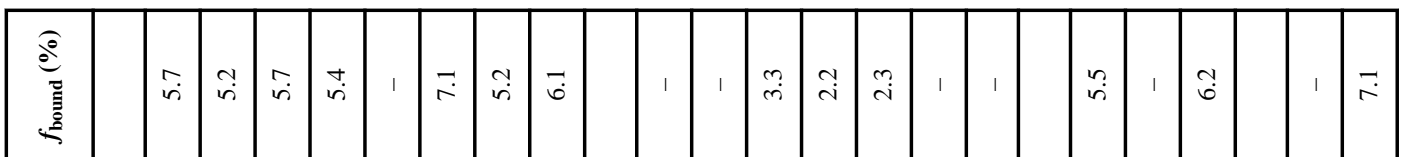

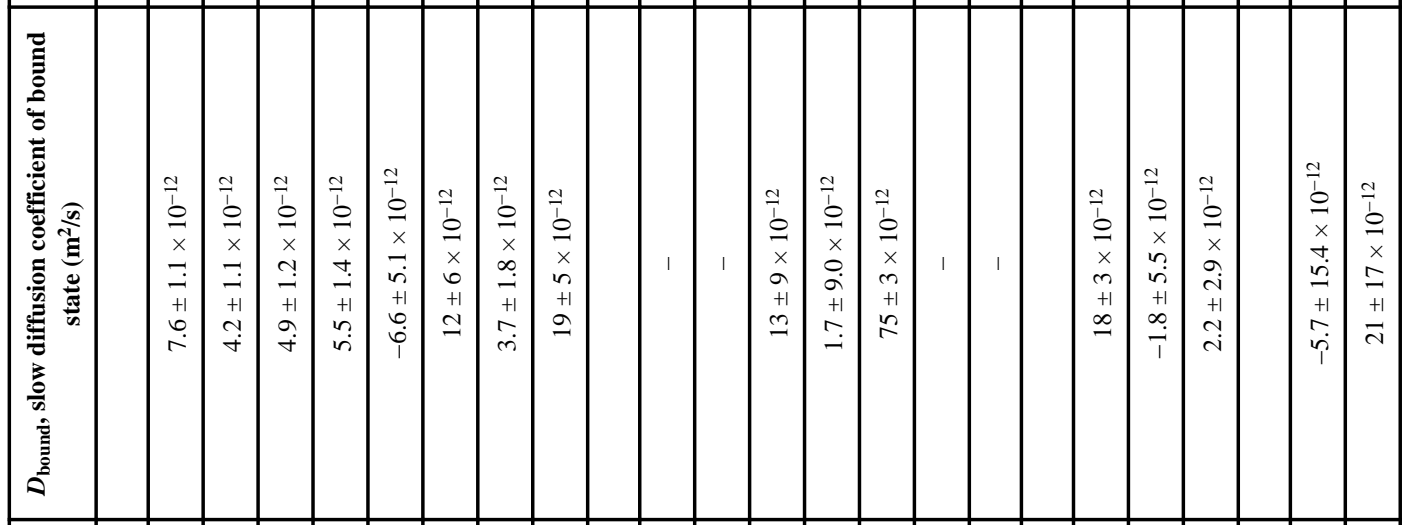

层

.

这

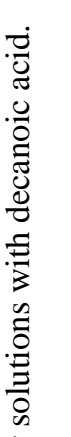

है

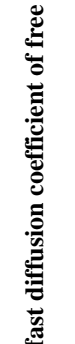

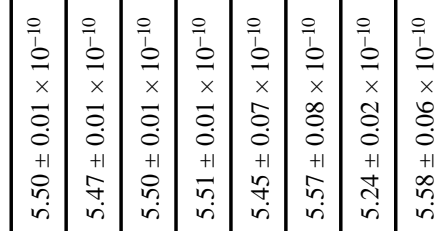

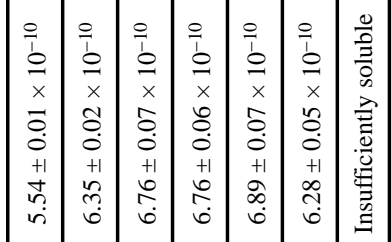

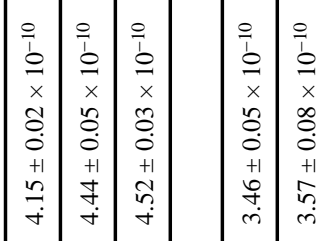

ग

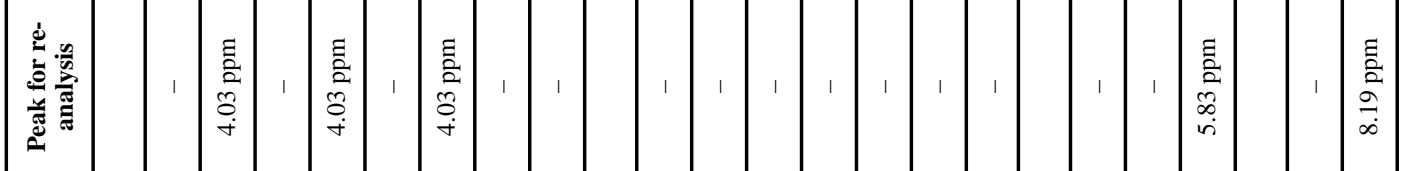

ग
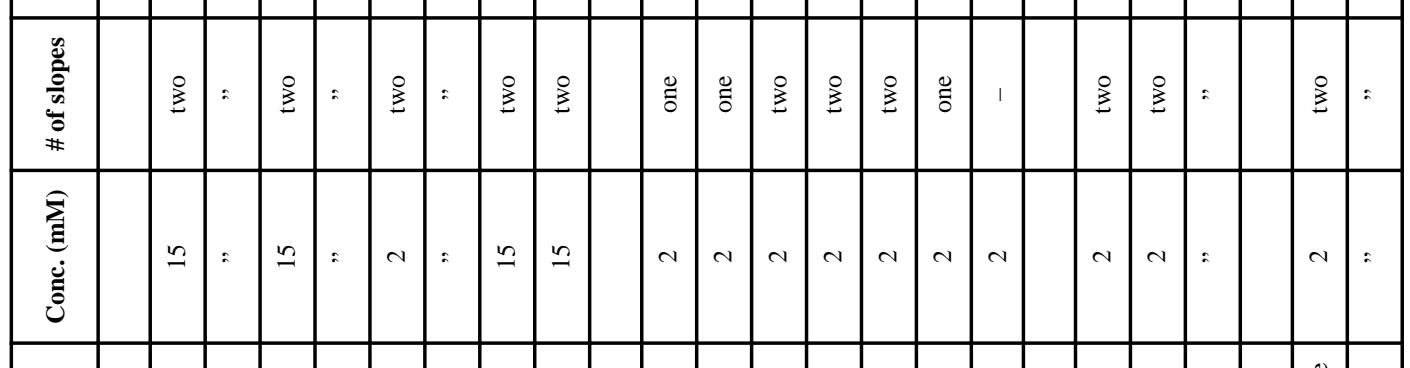

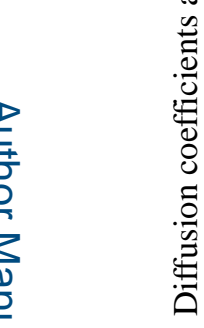

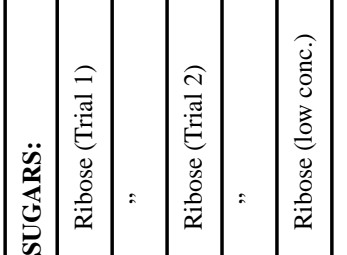

胥

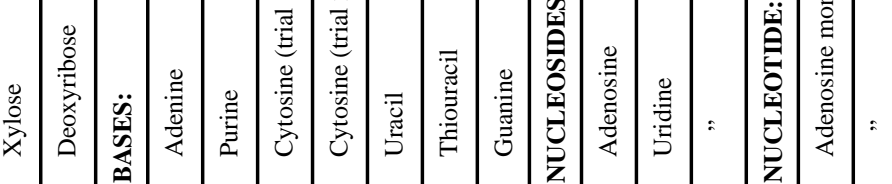

Chembiochem. Author manuscript; available in PMC 2021 October 01. 